\title{
Etat biologique du sédiment de la lagune Aghien à partir de l'Indice Malacologique de qualité des systèmes Lacustres (IMOL) (Sud-est, Côte d'Ivoire)
}

\author{
Jean-Renaud ALLOUKO*, Kinanpara KONE et Kotchi Yves BONY \\ UFR de l'environnement, Université Jean Lorougnon Guédé, BP 150 Daloa, Côte d'Tvoire. \\ *Auteur correspondant; E-mail: aljeanreno@yahoo.fr; Tel: +2250708508358
}

\begin{abstract}
Received: 22-05-2021
Accepted: 01-12-2021

Published: $31-21-2021$

\section{RESUME}

La lagune Aghien est une lagune périurbaine sujette à plusieurs types de pressions anthropiques. Ces pressions pourraient avoir des répercussions sur les communautés biologiques qui peuvent être des indicateurs de l'état de qualité écologique de cette lagune. Parmi ces communautés biologiques, il y a les mollusques qui sont potentiellement capables de coloniser la zone profonde des systèmes lagunaires et lacustres. L'objectif de cette étude était d'évaluer l'état biologique du sédiment de la lagune Aghien à partir de l'Indice Malacologique de qualité des systèmes Lacustres (IMOL). Sur chaque station, l'échantillonnage du sédiment, a été réalisé en raison de trois échantillons de sédiments prélevés avec la benne Van Veen. Au total 10 taxons appartenant à 7 genres de mollusques ont été collectés dans le sédiment des différentes stations de la lagune Aghien. Selon l'IMOL, cinq stations sur les onze indiquent un état biologique moyen. L'état biologique du sédiment est connu et présente un état biologique globalement moyen. L'IMOL peut être utilisé pour évaluer les eaux lentiques ayant 10 mètres de profondeur maximale.

(C) 2021 International Formulae Group. All rights reserved.
\end{abstract}

Mots clés: Etat biologique, sédiment, lagune Aghien, Indice IMOL, Côte d'Ivoire

\section{Biological state of the sediment of the Aghien lagoon from the Malacological Quality Index of Lacustrine Systems (IMOL) (South-east, Ivory Coast)}

\section{ABSTRACT}

The Aghien lagoon is a peri-urban lagoon subject to several types of anthropogenic pressures. These pressures could have effects on biological communities which may be ecological quality status indicators. Among these biological communities, mollusc's which are likely to colonize the deep zone of lagoon and lake systems. This study was carried out to assess the biological state of Aghien lagoon sediment from the malacological index of lake systems (IMOL). At each station, sediment sampling was performed due to three sediment samples taken with the Van Veen grab. A total of 10 taxa belonging to 7 kinds of molluscs were collected in the sediment of the various stations. According to IMOL, five of the eleven stations, had an average biological state. The biological state of the sediment was known and had an average biological state. IMOL can be used to assess lentic waters up to 10 meters deep.

(C) 2021 International Formulae Group. All rights reserved.

Keywords: Biological state, sediment, Aghien lagoon, IMOL index, Côte d'Ivoire. 


\section{INTRODUCTION}

La lagune Aghien est une lagune périurbaine sujette à plusieurs types de pressions anthropiques (Yao, 2020). Ces pressions pourraient avoir des répercussions sur les communautés biologiques qui peuvent être des indicateurs de l'état de qualité écologique de cette lagune (Humbert, 2012). Parmi ces communautés biologiques, il y a les mollusques qui sont potentiellement capables de coloniser la zone profonde des systèmes lagunaires et lacustres. Lorsque le milieu se dégrade (installation puis développement de conditions hypoxiques et accumulation de matière organique dans ses sédiments profonds) on observe une diminution de plus en plus importante du nombre $d$ ' espèces selon les profondeurs, ainsi qu'une disparition progressive de la zone profonde à la zone littorale des Gastéropodes puis des Bivalves (Mouthon, 1993). Ainsi, différents états biologiques peuvent être observés. Compte tenu du fait qu'il n'existe pas de données sur la tolérance ou l'intolérance des taxons à la pollution pour la Côte d'Ivoire, l'Indice Malacologique de qualité des systèmes Lacustres (IMOL) qui vise à évaluer l'état biologique global d'un plan d'eau naturelle (lac, lagune) de profondeur maximale compris entre 10 à 30 mètres (Mouthon, 1993 ; Barbe et al., 2003) a été utilisé. Les scores de sensibilité de cet indice ont été utilisés dans cette étude à cause de la nature et de la profondeur maximale de la lagune Aghien (10 mètres). L'objectif de cette étude est d'évaluer l'état biologique du sédiment de lagune Aghien à de l'IMOL.

\section{MATERIEL ET METHODE \\ Site d'étude}

Localisée entre les latitudes $5^{\circ} 22^{\prime} \mathrm{N}$ et $5^{\circ} 26^{\prime} \mathrm{N}$ et les longitudes $3^{\circ} 49^{\prime} \mathrm{W}$ et $3^{\circ} 55^{\prime} \mathrm{W}$, la lagune Aghien est située au Nord de la lagune Ebrié de laquelle elle est séparée par la lagune Potou (Figure 1) (Traoré et al., 2012). La lagune Aghien couvre un bassin versant de $340 \mathrm{~km} 2$ et s'étend sur $19 \mathrm{~km} 2$. Les lagunes Aghien et Potou communiquent par un chenal naturel. Les plus importants affluents de la lagune Aghien sont les rivières Bété, Djibi et Mé (Figure 1). La Bété et la Djibi débouchent directement dans la lagune Aghien tandis que la Mé se déverse dans le canal naturel entre les lagunes Aghien et Potou. Le système lagunaire Aghien-Potou s'étend sur $72 \mathrm{~km}$ de périmètre et $32 \mathrm{~km}$ de longueur de l'axe médian (Konan et al., 2017). La lagune Aghien peut atteindre $11 \mathrm{~m}$ de profondeur. Dans cette lagune d'eau douce, l'eau est toute l'année quasi exclusivement d'origine continentale (Humbert, 2012; Konan et al., 2017).

\section{Méthode d'échantillonnage}

Les sites d'échantillonnage ont été sélectionnés pour couvrir une bonne hétérogénéité de l'habitat dans la lagune d'Aghien. Les stations d'études sont situées le long de l'axe principal de la lagune avec deux transects perpendiculaires au transect longitudinal (Figure 2). Le premier transect était situé en face de la ville d'Akandjé afin de déterminer si la qualité de l'eau varie en fonction de la distance au rivage. Le deuxième transect était situé dans la zone Est de la lagune notamment entre les villages Aghien et Télégraphe. Des stations ont été retenues plus proche des rivières Djibi, Bété et Mé pour évaluer le taux d'échange des différents milieux (Figure 2). L'échantillonnage des mollusques a été réalisé pendant douze mois à intervalles mensuels entre juin 2014 et mai 2015.

Les mollusques ont été récoltés à l'aide d'une benne Van Veen en acier inoxydable. A chaque site, trois (03) échantillons de sédiment correspondant à une surface totale de $0,15 \mathrm{~m}^{2}$ ont été prélevés à plusieurs profondeurs. A la sortie de l'eau, le contenu de la benne a été lavé sur un tamis de $0,5 \mathrm{~mm}$ de maille. Tous les échantillons ont été fixés à l'alcool à $70 \%$. Au laboratoire, tous les échantillons obtenus ont été tamisés, et les individus récoltés, triés à l'aide d'une loupe 
binoculaire, dénombrés et identifiés au niveau taxonomique le plus bas possible en combinant des clés de détermination appropriées (Brown, 2005 ; Forcellini et al., 2011).

\section{Analyse des données}

\section{Mode de détermination de l'IMOL}

Pour chaque station d'échantillonnage, l'IMOL a été calculé à partir de la liste de mollusques du sédiment collectés. L'IMOL d'une station est obtenu en tenant compte du score attribué aux mollusques présents à une profondeur donnée. Le Tableau 1 ci-dessous présente le mode détermination de l'IMOL (Barbe et al., 2003).

\section{Classes de qualité de l'IMOL}

Les valeurs de l'indice IMOL déterminées correspondent à des classes de qualités biologiques des eaux proposées par l'Office National de l'eau et des milieux aquatiques (ONEMA) afin d'évaluer l'état biologique de l'eau (ONEMA, 2011). Ces classes de qualités sont consignées dans le Tableau 2.

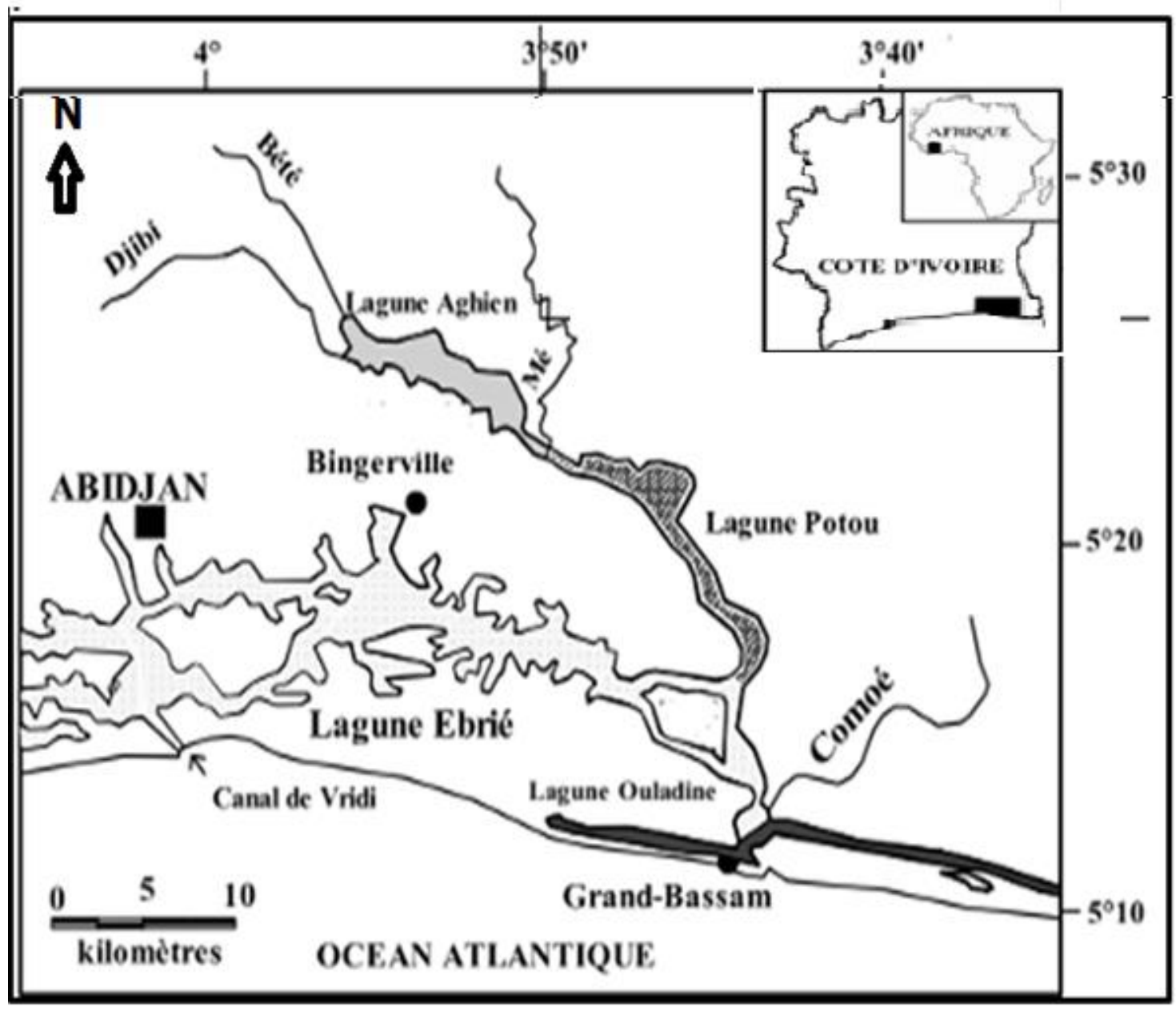

Figure 1: Localisation de la lagune Aghien au sud de la Côte d'Ivoire (Bedia et al., 2009). 


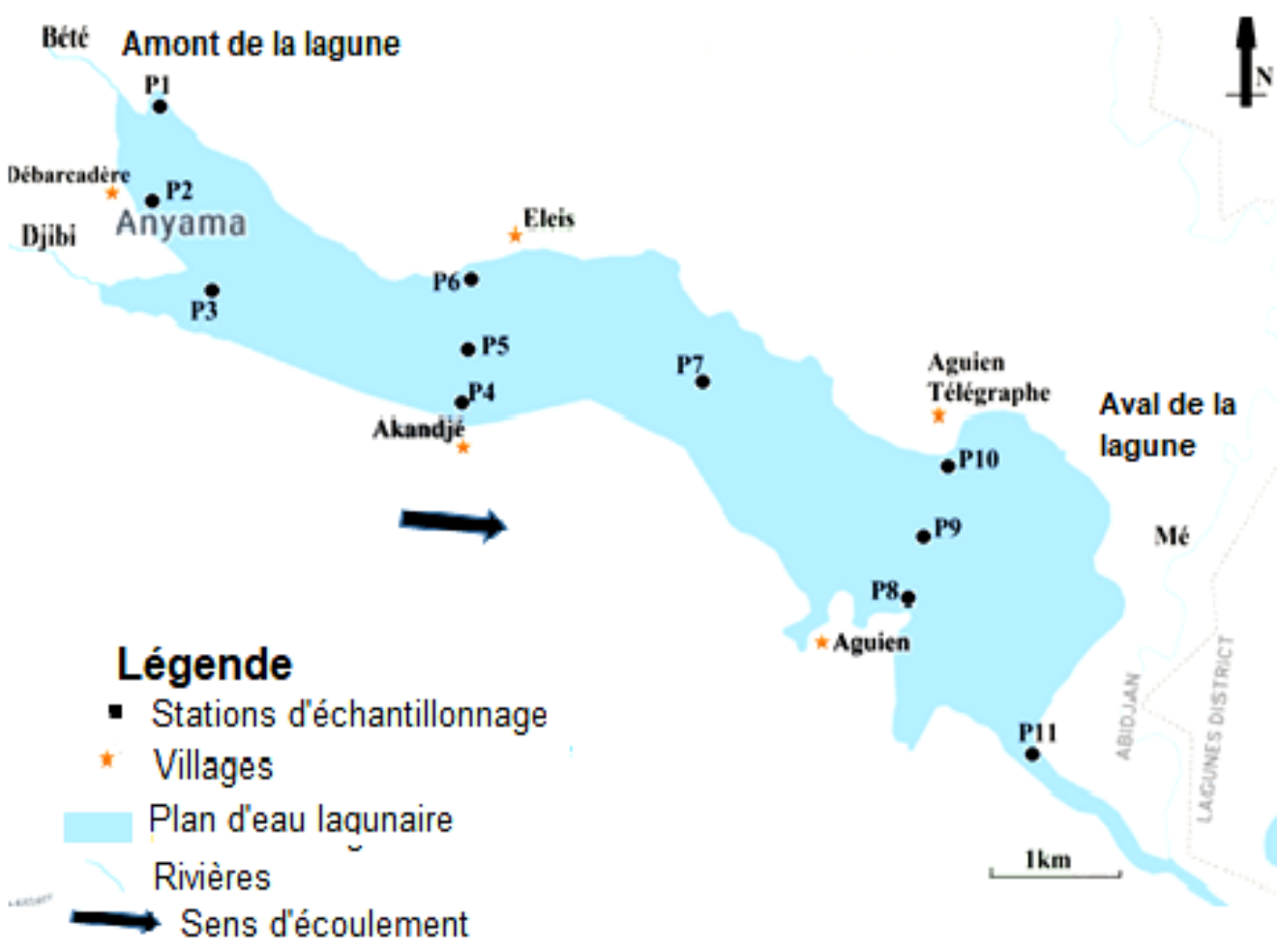

Figure 2 : Plan de la lagune Aghien et situation de sites d'échantillonnage (Humbert, 2012).

Tableau 1 : Détermination de l'IMOL (Barbe et al., 2003).

\begin{tabular}{|c|c|c|}
\hline $\begin{array}{l}\text { Profondeur } \\
\text { d'échantillonnage }\end{array}$ & Repères malacologiques & Indices \\
\hline \multirow{2}{*}{$\mathrm{Z1}=$ 9/10 Zmax } & Gastéropodes et Bivalves présents & 8 \\
\hline & Gastéropodes absents, Bivalves seuls présents & 7 \\
\hline \multirow{3}{*}{$Z 2=-10 \mathrm{~m}$} & $\begin{array}{l}\text { Deux genres ou plus de genres de } \\
\text { Gastéropodes présents }\end{array}$ & 6 \\
\hline & Un seul genre de Gastéropode présent & 5 \\
\hline & Gastéropodes absents, pisidies présents & 4 \\
\hline \multirow{4}{*}{$\mathbf{Z 3}=-\mathbf{3} \mathbf{~ m}$} & $\begin{array}{l}\text { Deux genres ou plus de deux genres de } \\
\text { Gastéropodes présents }\end{array}$ & 3 \\
\hline & Un seul genre de Gastéropode présent & 2 \\
\hline & Gastéropodes absents, pisidies présents & 1 \\
\hline & Absence de mollusques & 0 \\
\hline
\end{tabular}

$\mathbf{Z 1}$ à Z3 = profondeurs des différents sites d'échantillonnage ; $\boldsymbol{m}=$ mètre ; $\boldsymbol{m a x}=$ maximum 
Tableau 2 : Classes de qualité de l'IMOL (ONEMA, 2011).

\section{Limites des classes d'états \\ (Indices IMOL)}

IMOL $\geq 8$

$7 \leq \mathrm{IMOL}<8$

$4 \leq \mathrm{IMOL}<7$

$1 \leq \mathrm{IMOL}<4$

0

\section{Classes de qualité}

Très bonne

Bonne

Moyenne

Médiocre

Mauvaise

\section{RESULTATS}

Inventaire des mollusques du sédiment de la lagune Aghien

Le Tableau 3 présente le récapitulatif de la présence/absence des mollusques présents dans le sédiment de la lagune Aghien. Au total 10 taxons ont été collectés dans le sédiment des différentes stations de la lagune Aghien. Il y a un taxon appartenant à la classe des Bivalves et 09 taxons pour les Gastéropodes. Melanö̈des tuberculata et Potadoma liricincta sont respectivement présents dans 09 stations et 08 stations d'échantillonnage. Bulinus guernei et Bulinus truncatus ont été uniquement récoltés à la station P6.

\section{Etat biologique à partir de l'IMOL}

Le Tableau 4 présente les variations de l'indice IMOL et des états biologiques des sédiments des différentes stations d'échantillonnage. Les stations P1, P3, P8, ont chacune une valeur de l'IIMOL égale à 6 alors que les valeurs de 4 et 5 ont été enregistrées au niveau des stations P5 et P11. Les valeurs de l'IMOL de ces stations indiquent un état biologique moyen.

Les stations P7 et P9 ont respectivement une valeur égale 7. Les valeurs de l'IMOL de ces stations indiquent un Bon état biologique. Les stations P2, P4, P6 et P10 ont enregistré respectivement une valeur de l'indice IMOL égale à $3, \quad 2, \quad 3$ et 3 . Ces valeurs indiquent un état médiocre de ces stations. 
Tableau 3 : Mollusques du sédiment des stations d'échantillonnage.

\begin{tabular}{|c|c|c|c|c|c|c|c|c|c|c|c|c|}
\hline & & \multicolumn{11}{|c|}{ Station d'échantillonnage et Profondeur (m) } \\
\hline & & P1 & $\mathbf{P 2}$ & $\mathbf{P 3}$ & $\mathbf{P 4}$ & P5 & P6 & P7 & P8 & P9 & $\mathbf{P 1 0}$ & P11 \\
\hline Classe & Taxon & 3 & 1 & 6 & 2 & 9 & 1 & 10 & 3 & 10 & 1 & 5 \\
\hline \multirow[t]{5}{*}{ Bivalves } & Musculium sp. & & & + & & & & + & + & & + & + \\
\hline & Septaria borbonica & & & & & & & & + & & + & + \\
\hline & Bulinus guernei & & & & & & + & & & & & \\
\hline & Bulinus truncatus & & & & & & + & & & & & \\
\hline & Indoplanorbis exustus & + & & & + & + & & & & & & \\
\hline \multirow[t]{5}{*}{ Gastéropodes } & Melanoïdes tuberculata & + & + & + & + & & & + & + & + & + & + \\
\hline & Pachymelania byronensis & & & & & & + & + & + & + & + & + \\
\hline & Pachymelania fusca & & & & & & & + & + & + & + & + \\
\hline & Potadoma liricincta & + & & + & & & + & + & + & + & + & + \\
\hline & Septaria borbonica & & & & & & & & & & & + \\
\hline
\end{tabular}

P1 à P11 : Stations d'échantillonnage ; + : présence du taxon

Tableau 4 : Variations spatiales de l'indice IMOL et des états biologiques.

\begin{tabular}{lcl}
\hline Stations & Indice IMOL & Etat biologique \\
\hline P1 & 6 & Moyen \\
P2 & 3 & Médiocre \\
P3 & 6 & Moyen \\
P4 & 2 & Médiocre \\
P5 & 4 & Moyen \\
P6 & 3 & Médiocre \\
P7 & 7 & Bon \\
P8 & 6 & Moyen \\
P9 & 7 & Bon \\
P10 & 3 & Médiocre \\
P11 & 5 & Moyen \\
\hline
\end{tabular}




\section{DISCUSSION}

L'utilisation de l'IMOL qui vise à évaluer l'état biologique global d'un plan d'eau naturel (lac, lagune) de profondeur maximale comprise entre 10 à 30 mètre a été faite avec succès sur la lagune Aghien qui a une profondeur maximale de 10 mètres (Barbe et al., 2003). En effet, au total 10 taxons ont été collectés dans le sédiment des différentes stations de la lagune Aghien avec une dominance des Gastéropodes (09 taxons). Ceci pourrait s'expliquer d'une part, par la disponibilité de conditions favorables au développement des taxons indicateurs polluoresistants tels que les Thiaridae et la relative faible profondeur de certaines zones d'échantillonnage d'autre part (Barbe et al., 2003).

L'IMOL dans les stations varie entre 2 et 7 , valeurs indiquant respectivement un état médiocre et bon. La moitié des stations proches des berges ont des valeurs de l'IMOL plus faibles indiquant un état biologique médiocre de l'eau. Ce résultat découlerait de la relative faible profondeur de certaines zones d'échantillonnage qui sont proches des villages (Débarcadère; Akandjé et Télégraphe) où il y a une forte pression des activités anthropique sur les communautés biologiques. Les fortes valeurs de l'IMOL enregistrées au niveau des stations situées en pleine eau peuvent s'expliquer par les compositions taxonomiques des peuplements benthiques de la lagune Aghien notamment la présence de taxons de Bivalves et de Gastéropodes dans le sédiment (ONEMA, 2011). Par ailleurs, les valeurs élevées de l'IMOL étaient associées aux stations avec de faibles pressions directes sur les communautés biologiques compte tenue de la distance avec les zones activités anthropiques.

Dans l'ensemble, sur onze stations, deux stations ont présenté un bon état biologique pendant que cinq stations ont présenté un état moyen. Au regard des résultats, le sédiment de la lagune Aghien présenterai un état biologique moyen.

Cependant, des macroinvertébrés reconnus comme étant des vecteurs ou hôtes potentiels des pathogènes de certaines maladies comme l'ulcère de Burili et la Bilharziose ont été inventoriés. Il s'agit d'Indoplanorbis exustus.

\section{Conclusion}

L'état biologique du sédiment de la lagune Aghien a été évalué avec succès à partir des 10 taxons des mollusques collectés. Cette lagune présente un état biologique globalement moyen. L'IMOL pourrait être utilisé pour évaluer les états biologiques des eaux lentiques des systèmes lagunaires ivoirien ayant 10 mètres de profondeur maximale. La présence de taxon tel qu'Indoplanorbis exustus implique que cette lagune devrait faire l'objet d'une biosurveillance.

\section{CONFLIT D'INTÉRÊTS}

Les auteurs déclarent ne pas avoir de conflits d'intérêts.

\section{CONTRIBUTIONS DES AUTEURS}

JRA a effectué les travaux de terrain, analysé les données et rédigé le premier draft de l'article. KK a contribué à l'interprétation des résultats et à la finition du manuscrit. KYB a conçu le projet et élaboré le protocole expérimental.

\section{REMERCIEMENTS}

Pour la réalisation de ce travail, nous avons bénéficié des données collectées dans le cadre du projet «évaluation de la qualité de l'eau de la lagune Aghien ». Nous remercions les promoteurs ce projet, notamment: l'Agence Française pour le Développement (AFD), l'Université Jean Lorougnon Guédé et le Ministère des Eaux et Forêts de Côte d'Ivoire. 


\section{REFERENCES}

Barbe J, Lafont M, Mouthon J, Philippe M. 2003. Protocole actualisé de la diagnose rapide des plans d'eau. Cemagref, Lyon, p. 30

Bedia AT, N'Zi KG, Yao SS, Kouamelan EP, N'Douba V, Kouassi NJ. 2009. Typologie de la pêche en lagune AghienPotou (Côte d'Ivoire, Afrique de l'ouest) : acteurs et engins de pêche. Agron. Afr., 21(2): 197-204. DOI: 10.4314/aga.v21i 2.49810

Brown DS. 2005 Freshwater Snails of Africa and Their Medical Importance (2nd edn) Taylor \& Francis: London, 673 p.

Forcellini M, Mathieu C, Merigoux S. 2011. Atlas des Macroinvertébrés des eaux douces de l'île de la Réunion. Office de l'eau de la réunion, CNRS ; $137 \mathrm{p}$.

Humbert JF. 2012. Rapport sur la mission « lagune Aghien ». Rapport d'expertise, AFD-MINEF-SODECI-C2D, $25 \mathrm{p}$.

Konan SK, Ko uakou BK, Ohou MJ, Konan FK, Dongui KB. 2017. Variation saisonnière des paramètres abiotiques de la lagune Aghien (Côte d'Ivoire). $J$. Appl. Biosci., 120: 12042-12052. DOI: https://dx.doi.org/10.4314/jab.v120i1.7

ONEMA. 2011. Suivi des plans d'eau des bassins Rhône Méditerranée et Corse en application de la Directive Cadre sur l'Eau. Note synthétique d'interprétation des résultats, Office National de l'eau et des milieux aquatiques, France, $19 \mathrm{p}$.

Traoré A, Soro G, Kouadio KE, Bamba SB, Oga MS, Soro N, Biémie J. 2012. Evaluation des paramètres physiques, chimiques et bactériologiques des eaux d'une lagune tropicale en période d'étiage : la lagune Aghien (Côte d'Ivoire). Int. J. Biol. Chem. Sci., 6(6): 7048-7058.

DOI http://dx.doi.org/10.4314/ijbcs.v6i6.40

Yao DAR. 2020. Etude des cyanobactéries de la lagune Aghien et de leur potentialité à produire des métabolites secondaires. Thèse de doctorat, Université Félix Houphouët-Boigny, Abidjan, Côte d'Ivoire, $191 \mathrm{p}$. 